\title{
INVERSE-CLOSED CARLEMAN ALGEBRAS OF INFINITELY DIFFERENTIABLE FUNCTIONS
}

\author{
JAMIL A. SIDDIQI \\ (Communicated by John B. Conway)
}

\begin{abstract}
We characterize the classes $\mathscr{C}_{M}(I)$ and $\mathscr{C}_{M}^{*}(I)$ of infinitely differentiable functions which are inverse-closed thereby giving a complete solution to a problem first posed by W. Rudin [11] and solved by him and J. Boman and L. Hörmander [2] for classes $\mathscr{C}_{M}(\mathbf{R})$ alone.
\end{abstract}

\section{INTRODUCTION}

Given a positive sequence $M=\left\{M_{n}\right\}$ and an interval $I$, let $\mathscr{C}_{M}(I)$ denote the Carleman class of all infinitely differentiable complex functions $f$ defined on $I$ for which $\sup _{n}\left\{\left\|f^{(n)}\right\|_{\infty} / M_{n}\right\}^{1 / n}<\infty$. A class $\mathscr{C}_{M}(I)$ is said to be inverseclosed if $f^{-1}$ is in $\mathscr{C}_{M}(I)$ whenever $f$ is in $\mathscr{C}_{M}(I)$ and is bounded away from zero. More generally, analytic functions are said to operate on $\mathscr{C}_{M}(I)$ if for any $f$ in $\mathscr{C}_{M}(I)$ and for any $g$ analytic in an open set containing the closure of the range of $f, g \circ f$ is in $\mathscr{C}_{M}(I)$. A sequence $M$ is called log-convex if $M_{n}^{2} \leq M_{n-1} M_{n+1}$ for $n \geq 1$.

In his paper on the symbolic calculus for the algebra of real functions which are Fourier transforms of functions in $L^{1}(G)$, P. Malliavin [8] (see J.-P. Kahane [7] for this and other related results) proved the following:

Theorem A. If $M$ is a log-convex sequence and the sequence $A \equiv\left\{A_{n}=\right.$ $\left.\left(M_{n} / n !\right)^{1 / n}\right\}$ is almost increasing in the sense that there exists a constant $K>0$ such that $A_{m} \leq K A_{n}$ for each $m$ and $n$ with $m<n$, then the class $\mathscr{C}_{M}(I)$ is inverse-closed.

The problem as to whether the converse of Theorem A holds was first taken up by W. Rudin [11] who proved that it is so if $\mathscr{C}_{M}(R)$ is a non-quasi-analytic class of $2 \pi$-periodic functions, a restriction that was later removed by J. Boman and

Received by the editors May 4, 1989.

1980 Mathematics Subject Classification (1985 Revision). Primary 46E25; Secondary 30D60.

Key words and phrases. Carleman and local Carleman classes, inverse-closed algebras, log-convex sequences, almost increasing sequences.

This research was supported by NSERC of Canada and FCAR of the Ministry of Education, Québec. 
L Hörmander [2]. Thus even when the Carleman classes on $I \neq \mathbf{R}$ are defined by log-convex sequences, the converse of Theorem $\mathrm{A}$ is not known.

It is clear that Malliavin's sufficiency condition is applicable only to classes $\mathscr{C}_{M}(I)$ which are defined by log-convex sequences. However there exist classes $\mathscr{C}_{M}(I)$ which cannot be so defined. In fact, H. Cartan [5] has shown that if $I$ is closed, then $\mathscr{C}_{M}(I) \not \equiv \mathscr{C}_{M^{c}}(I)$ even if $\mathscr{C}_{n !}(I) \subseteq \mathscr{C}_{M}(I)$, where $M^{c}$ denotes the largest log-convex minorant of $M$. Thus for classes $\mathscr{C}_{M}(I)$ not defined by log-convex sequences, the problem of finding necessary and sufficient condition on $M$ in order that they be inverse-closed over arbitrary $I$, has remained open so far (see, however, [12]). The same holds for local Carleman classes $\mathscr{C}_{M}^{*}(I)$ of functions which belong to class $\mathscr{C}_{M}(J)$ for each compact subinterval $J$ of $I$.

In this paper, we give a complete solution of the problem for these classes. Instead of limiting a priori to classes defined by log-convex sequences $M$, we consider arbitrary classes which, as is well known (see [10]), can also be defined by regularized sequences $M^{r}$ which vary according to the nature of the interval $I$. Using either the characteristic functions of these classes or Baire's category theorem applied to certain Fréchet spaces, we are able to show that they are inverse-closed if and only if $\left\{\left(M_{n}^{r} / n !\right)^{1 / n}\right\}$ is almost increasing. The techniques employed here are different. They are simpler than those used for $I=\mathbf{R}$ in [11] and [2] and enable us to solve, in particular, the problem of characterization of inverse-closed algebras $\mathscr{C}_{M}^{2 \pi}([-\pi, \pi])$ of $2 \pi$-periodic functions, posed by W. Rudin in [11].

The inverse-closed algebras $\mathscr{C}_{M}(I)$ and $\mathscr{C}_{M}^{*}(I)$ are, respectively, inductive limits of Banach and Fréchet spaces. Although, with the usual seminorms, they are not locally convex algebras (see [9] for relevant definitions), we can describe their (algebraic) maximal ideals and complex homomorphisms. Thus every maximal ideal of the inverse-closed algebra $\mathscr{C}_{M}(I)$ is of the form $\mathscr{I}_{x}=$ $\left\{f \in \mathscr{C}_{M}(I): f(x)=0\right\}$ for some $x \in I$ and every complex homeomorphism is a point evaluation.

We may remark that $\mathrm{J}$. Bruna [3] has proved that if $M$ is log-convex, then the differentiable Beurling classes and their projective limits are inverse-closed if and only if $\left\{\left(M_{n} / n !\right)^{1 / n}\right\}$ is almost increasing.

\section{INVERSE-Closed CARLEMAN ClASSES}

A Carleman class $X=\mathscr{C}_{M}(\mathbf{R})$ is always an algebra. In fact, if

$$
\liminf _{n \rightarrow \infty} M_{n}^{1 / n}=0, \quad X \equiv\{\text { const }\}
$$

and if

$$
0<\liminf _{n \rightarrow \infty} M_{n}^{1 / n}<\infty, \quad X \equiv \mathscr{C}_{1}(\mathbf{R})
$$

In both these cases $X$ is an algebra. Suppose now that $\lim _{n \rightarrow \infty} M_{n}^{1 / n}=\infty$. If 
we set

then

$$
T_{M}(r)=\sup _{n \geq 1} \frac{r^{n}}{M_{n}}
$$

$$
M_{n}^{c}=\sup _{r \geq 1} \frac{r^{n}}{T_{s c}(r)}, \quad T_{s c}(r)=\sup _{n \geq 1} \frac{r^{n}}{M_{n}^{c}}=T_{M^{c}}(r) .
$$

Since $X \equiv \mathscr{C}_{M^{c}}(\mathbf{R})$ (see [10], p. 226), and $M^{c}$ is log-convex, using the Leibnitz formula for successive derivatives of a product, it is easily seen that $X$ is an algebra.

Similarly $X=\mathscr{C}_{M}\left(\mathbf{R}^{+}\right)$is always an algebra. In fact, if $\liminf _{n \rightarrow \infty} n M_{n}^{1 / n}=$ $0, X \equiv\{$ const $\}$ and if $0<\liminf _{n \rightarrow \infty} n M_{n}^{1 / n}<\infty$ then $X \equiv \mathscr{C}_{n^{-n}}\left(\mathbf{R}_{+}\right)$. Suppose now that $\lim _{n \rightarrow \infty} n M_{n}^{1 / n}=\infty$. Then $X \equiv \mathscr{C}_{M^{d}}\left(\mathbf{R}_{+}\right)$(see [10], p. 226), where $M^{d} \equiv\left\{M_{n}^{d}\right\}$ is defined by setting $n^{n} M_{n}^{d}=\left(n^{n} M_{n}\right)^{c} \quad(n \geq 1)$. If we put

$$
T_{M}^{*}(r)=\sup _{n \geq 1} \frac{r^{n}}{n^{n} M_{n}} \quad(r \geq 1)
$$

then

$$
n^{n} M_{n}^{d}=\sup _{r \geq 1} \frac{r^{n}}{T_{s c}^{*}(r)} \quad \text { and } \quad T_{s c}^{*}(r)=\sup _{n \geq 1} \frac{r^{n}}{n^{n} M_{n}^{d}}=T_{M^{d}}^{*}(r) .
$$

Here, as before, in all the above three cases $\mathscr{C}_{M}\left(\mathbf{R}_{+}\right)$is an algebra.

A Carleman class $\mathscr{C}_{M}(I)$ consisting of the constants alone is always inverseclosed. For nontrivial inverse-closed Carleman classes, we have the following characterization.

Theorem 1. Let $X=\mathscr{C}_{M}(\mathbf{R})$ or $\mathscr{C}_{M}\left(\mathbf{R}_{+}\right)$. If $X$ is nontrivial, then the following assertions are equivalent:

(a) $\lim _{n \rightarrow \infty} M_{n}^{1 / n}=\infty$ and the sequence $A$ is almost increasing.

(b) Analytic functions operate on $X$.

(c) $X$ is an inverse-closed algebra.

Here $A=\left\{A_{n}\right\}$, where $A_{n}=\left(M_{n}^{c} / n !\right)^{1 / n}$ or $\left(M_{n}^{d} / n !\right)^{1 / n} \quad(n \geq 1)$ according as $X=\mathscr{C}_{M}(\mathbf{R})$ or $\mathscr{C}_{M}\left(\mathbf{R}_{+}\right)$.

Proof. (i) Let $X=\mathscr{C}_{M}(\mathbf{R})$. Suppose that (a) holds. Then $X \equiv \mathscr{C}_{M^{c}}(\mathbf{R})$. That analytic functions operate on $X$ now follows since $A$ is almost increasing, if we use Faà di Bruno's formula viz.,

$$
(g \circ f)^{(n)}(x)=\sum \frac{n !}{k_{1} ! \cdots k_{n} !} g^{(k)}(f(x))\left(\frac{f^{\prime}(x)}{1 !}\right)^{k_{1}} \cdots\left(\frac{f^{(n)}(x)}{n !}\right)^{k_{n}},
$$

where

$$
2^{n-1}=\sum \frac{k !}{k_{1} ! \cdots k_{n} !},
$$

and the summation in the two cases is over all $n$-tuples $\left(k_{1}, \ldots, k_{n}\right)$ such that $k_{1}+\cdots+k_{n}=k$ and $k_{1}+2 k_{2}+\cdots+n k_{n}=n(0 \leq k \leq n)$. Thus (b) holds. 
Trivially (b) implies (c). It remains to be shown that (c) implies (a). Let (c) hold. Since $X$ is nontrivial, we cannot have $\liminf _{n \rightarrow \infty} M_{n}^{1 / n}=0$, for otherwise, we would have $\mathscr{C}_{M}(\mathbf{R}) \equiv$ const $\}$. We cannot have $0<\liminf _{n \rightarrow \infty} M_{n}^{1 / n}<\infty$ either, for then we would have $\mathscr{C}_{M}(\mathbf{R}) \equiv \mathscr{C}_{1}(\mathbf{R})$ which is not inverse-closed contradicting the hypothesis, since $f$, where $f(x)=2+\sin x$ is in $\mathscr{C}_{1}(\mathbf{R})$ but not its inverse (see [1], p. 25). Thus $\lim _{n \rightarrow \infty} M_{n}^{1 / n}=\infty$. It follows from (1) that there exists a positive sequence $\left\{r_{n}\right\}$ such that $r_{n}^{n}=M_{n}^{c} T_{M^{c}}\left(r_{n}\right) \quad(n \geq 1)$. The function

$$
f(x)=\sum_{j=1}^{\infty} \frac{e^{i r_{j} x}}{2^{j} T_{M^{c}}\left(r_{j}\right)},
$$

is a characteristic function of $X$ since it is easily seen that

$$
\begin{aligned}
& 1^{\circ} \cdot\left|f^{(n)}(x)\right| \leq M_{n}^{c}(n \geq 0 ; x \in \mathbf{R}), \\
& 2^{\circ} . f^{(n)}(0)=i^{n} s_{n}(n \geq 0), \text { where } s_{n} \geq 2^{-n} M_{n}^{c} .
\end{aligned}
$$

If $\lambda>1+\|f\|_{\infty}$, then $\lambda-f \in X$ and since it does not vanish on $\mathbf{R}$ and $X$ is inverse-closed, $(\lambda-f)^{-1}$ belongs to $X$. From (3), we get

$$
\sum \frac{k !}{k_{1} ! \cdots k_{n} !}(\lambda-f(0))^{-k-1}\left(\frac{M_{1}^{c}}{1 ! 2}\right)^{k_{1}} \cdots\left(\frac{M_{n}^{c}}{n ! 2^{n}}\right)^{k_{n}} \leq A B^{n} \frac{M_{n}^{c}}{n !} .
$$

Let $p>1$ be a fixed integer and suppose that $n=p k$. The term in the above inequality that corresponds to the choice $k_{p}=k$ and $k_{q}=0$ for $q \neq p$ does not exceed that on the right so that

$$
\left(\frac{M_{p}^{c}}{p !}\right)^{1 / p} \leq K_{1}\left(\frac{M_{n}^{c}}{n !}\right)^{1 / n}
$$

If $n$ is not a multiple of $p$, let $p m \leq n \leq p(m+1)$. Since $\left\{\left(M_{n}^{c}\right)^{1 / n}\right\}$ is increasing, we get

$$
\left(\frac{M_{n}^{c}}{n !}\right)^{1 / n} \geq\left(\frac{M_{p m}^{c}}{(p m) !}\right)^{1 / p m} \frac{((p m) !)^{1 / p m}}{(n !)^{1 / n}} \geq \frac{1}{K_{2}}\left(\frac{M_{p}^{c}}{p !}\right)^{1 / p}
$$

so that (a) holds.

(ii) Let $X=\mathscr{C}_{M}\left(\mathbf{R}_{+}\right)$. Suppose that (a) holds. Since $\lim _{n \rightarrow \infty} n M_{n}^{1 / n}=\infty$, $X \equiv \mathscr{C}_{M^{d}}\left(\mathbf{R}_{+}\right)$. As in (i), (a) implies (b) and (b) implies (c). Suppose that (c) holds. Since $X$ is nontrivial, we cannot have $\liminf _{n \rightarrow \infty} n M_{n}^{1 / n}=0$. We cannot have $0<\liminf _{n \rightarrow \infty} n M_{n}^{1 / n}<\infty$. For then $X \equiv \mathscr{C}_{n^{-n}}\left(\mathbf{R}_{+}\right)$which is not inverse-closed. In fact, let

$$
h(x)=\sum_{k=0}^{\infty} \frac{(-x)^{k}}{(2 k+3) !}, \quad\left(x \in \mathbf{R}_{+}\right) .
$$

Using the properties of Mittag-Leffler function, we prove that

$$
\left|h^{(n)}(x)\right| \leq 2 e^{n} n^{-n} \quad(n \geq 1), \quad\left(x \in \mathbf{R}_{+}\right),
$$


so that $h \in X$. Choosing $\lambda>1+\|h\|_{\infty}$ and applying (3), we get

$$
\left[(\lambda-h(x))^{-1}\right]_{x=0}^{(2 m)} \geq \frac{(2 m) !}{(7 !)^{m} m !(\lambda-h(0))^{m+1}} .
$$

It follows that

$$
\limsup _{n \rightarrow \infty} n\left(\max _{x \in \mathbf{R}_{+}}\left|\left\{(\lambda-h(x))^{-1}\right\}^{(n)}\right|\right)^{1 / n}=\infty .
$$

Hence $(\lambda-h)^{-1} \notin X$, i.e. $X$ is not inverse-closed contradicting the hypothesis. Thus $\lim _{n \rightarrow \infty} n M_{n}^{1 / n}=\infty$ and $X \equiv \mathscr{C}_{M^{d}}\left(\mathbf{R}_{+}\right)$. It follows from (2) that there exists a positive sequence $\left\{r_{n}\right\}$ such that $r_{n}^{n}=n^{n} M_{n}^{d} T_{M^{d}}^{*}\left(r_{n}\right) \quad(n \geq 1)$. The function

$$
f(x)=\sum_{j=1}^{\infty} \frac{h\left(r_{j} x\right)}{2^{j} T_{M^{d}}^{*}\left(r_{j}\right)}
$$

is a characteristic function of $X$ since, by (5),

$$
\begin{aligned}
& 1^{\circ} \cdot\left|f^{(n)}(x)\right| \leq 2 e^{n} M_{n}^{d} \quad\left(n \geq 0 ; x \in \mathbf{R}_{+}\right), \\
& 2^{\circ} . f^{(n)}(0)=(-1)^{n} s_{n} \text {, where } s_{n} \geq \mu^{n} M_{n}^{d} \quad(\mu>0) .
\end{aligned}
$$

If we choose $\lambda>1+\|f\|_{\infty}$, then reasoning as in (i), we conclude that if $p>1$ is a fixed integer and $n$ is a multiple of $p$, say, $n=p k$, we get

$$
\frac{1}{(\lambda-f(0))^{k+1}}\left(\frac{p^{p} p ! M_{p}^{d}}{(2 p+3) ! 2^{p}}\right)^{k} \leq A B^{n} \frac{M_{n}^{d}}{n !}
$$

and consequently

$$
\left(\frac{M_{p}^{d}}{p !}\right)^{1 / p} \leq K\left(\frac{M_{n}^{d}}{n !}\right)^{1 / n}
$$

The result for arbitrary $n$ now follows as in (i) if we remember that $\left\{\left(n^{n} M_{n}^{d}\right)^{\frac{1}{n}}\right\}$ is increasing.

\section{INVERSE-ClOSED local CARLEMAN ClASSES}

We now proceed to characterize the inverse-closed local Carleman classes.

If $I$ is a finite or infinite open interval, then $\mathscr{C}_{M}^{*}(I) \equiv \mathscr{C}_{M^{0}}^{*}(I)$ (see [10], p. 223), where $M^{0}=\left\{M_{n}^{0}\right\}$ is defined by setting

$$
S_{M}(r)=\max _{n \leq r} \frac{r^{n}}{M_{n}}, \quad M_{n}^{0}=\sup _{r \geq n} \frac{r^{n}}{S_{M}(r)} .
$$

Then $S_{M}(r)=S_{M^{0}}(r)$. But if $I$ is an arbitrary interval then $\mathscr{C}_{M}^{*}(I) \equiv \mathscr{C}_{M^{f}}^{*}(I)$ (see [10], p. 223 and [4], p. 718), where $M^{f}=\left\{M_{n}^{f}\right\}$ is defined by setting

$$
U_{s c}(r)=\max _{n \leq r} \frac{r^{2 n}}{n^{n} M_{n}} \quad \text { and } \quad n^{n} M_{n}^{f}=\sup _{r \geq n} \frac{r^{2 n}}{U_{M}(r)} .
$$

Then $U_{M}(r)=U_{M^{f}}(r)$. 
A local Carleman class $X=\mathscr{C}_{M}^{*}(I)$ is not always an algebra (see [6], p. 337). However, it is so if it contains the local analytic class. In fact, if $I$ is open, then, as shown by H. Cartan (see [5], p. 7), $X \equiv \mathscr{C}_{M^{c}}^{*}(I)$ so that $X \equiv \mathscr{C}_{M^{0}}^{*}(I) \equiv \mathscr{C}_{M^{c}}^{*}(I)$ is an algebra. The same is true for local Carleman class $\mathscr{C}_{M}^{*}(I)$ defined on a closed or a semi-closed interval $I$. However in this case the following analogue of Cartan's result holds.

Lemma I. If $X=\mathscr{C}_{M}^{*}(I) \supseteq \mathscr{C}_{n !}^{*}(I)$, then $X \equiv \mathscr{C}_{M^{f}}^{*}(I) \equiv \mathscr{C}_{M^{d}}^{*}(I)$ for any interval I. Consequently $X$ is an algebra.

Proof. Since $X \equiv \mathscr{C}_{M}^{*}(I) \supseteq \mathscr{C}_{n !}^{*}(I)$, it follows that $M_{n} \geq k^{n} M_{0} n !(n \geq 0)$. Choose $f \in \mathscr{C}_{M}^{*}(I)$. Let $J$ be a compact subinterval of $I$ and let $I^{\prime}$ be a compact subinterval of $I$ such that each point $x$ of $J$ is in a subinterval of fixed length $\lambda(<1)$ contained in $I^{\prime}$, where $\left|f^{(n)}(x)\right| \leq K \sigma^{n} M_{n} \quad(n \geq 0)$.

Choose $\sigma$ so large that $\lambda \sigma \geq k^{-1}$. Clearly $n ! k \quad M_{0} \leq K(\lambda \sigma)^{n} M_{n} \quad(n \geq 0)$. Let $\left\{n_{i}\right\}$ be the sequence of principal indices for $M^{\prime}=\left\{K \sigma^{n} M_{n}\right\}$ and let $n_{i}<$ $n<n_{i+1}$. Since $\left(K \sigma^{n} n^{n} M_{n}^{d}\right)$ is log-convex, applying Cartan-Gorny inequalities (see [10], p. 219), we conclude that for any $x$ in $J$

$$
\begin{aligned}
\left|f^{(n)}(x)\right| & \leq 2\left(e^{2} r p^{-1}\right)^{p}\left(K(\lambda \sigma)^{n_{i}} M_{n_{i}}\right)^{q / r}\left(K(\lambda \sigma)^{n_{i+1}} M_{n_{i+1}}\right)^{p / r} \\
& \leq 2 K\left(e^{2} r p^{-1}\right)^{p} n_{i}^{-n_{i} q / r} n_{i+1}^{-n_{i+1} p / r}(\lambda \sigma n)^{n} M_{n}^{d},
\end{aligned}
$$

where $p=n-n_{i}, q=n_{i+1}-n, r=n_{i+1}-n_{i}$. Set $u=n_{i+1} / n_{i}$. Then

$$
n_{i}^{-n_{i} q / r} n_{i+1}^{-n_{i+1} p / r}=\left(u^{u /(u-1)}(u-1)\right)^{n-n_{i}} \leq 1 \text {. }
$$

Hence

$$
\left|f^{(n)}(x)\right| \leq \mu^{n} M_{n}^{d} \quad(x \in J) .
$$

Since $M_{n_{i}}=M_{n_{i}}^{d}$, this inequality holds for all $n \geq 0$. Thus $f \in \mathscr{C}_{M^{d}}^{*}(I)$. Clearly $\mathscr{C}_{M^{d}}^{*}(I)$ is an algebra.

Thus a local Carleman algebra $X=\mathscr{C}_{M}^{*}(I) \supseteq \mathscr{C}_{n !}^{*}(I)$ has two regularizations viz. $X \equiv \mathscr{C}_{M^{0}}^{*}(I) \equiv \mathscr{C}_{M^{c}}^{*}(I)$ or $X \equiv \mathscr{C}_{M^{f}}^{*}(I) \equiv \mathscr{C}_{M^{d}}^{*}(I)$ according as $I$ is open or arbitrary.

Although, in general, it is not true, the equivalence of classes in these two cases does imply that the sequences $M^{0}$ and $M^{c}$ and the sequences $M^{f}$ and $M^{d}$ are equivalent in the sense that for some constants $\alpha>0$ and $\beta>0$

$$
\begin{aligned}
& \text { (a) } \beta^{n} M_{n}^{0} \leq M_{n}^{c} \leq \alpha^{n} M_{n}^{0} \text {, } \\
& \text { (b) } \beta^{n} M_{n}^{f} \leq M_{n}^{d} \leq \alpha^{n} M_{n}^{f} \quad(n \geq 1) \text {. }
\end{aligned}
$$

The second halves of (7)(a) and (b) are obviously true with $\alpha=1$. For $I$ finite, (7) follows from the inclusion theorems of H. Cartan and S. Mandelbrojt (see [10], p. 238). The arguments used by these authors fail when $I$ is infinite. However, using Baire's category theorem, we get the same result valid in all cases.

Lemma II. For any finite or infinite open interval $I, \mathscr{C}_{M}^{*}(I) \subseteq \mathscr{C}_{N}^{*}(I)$ if and only if $\left(M_{n}^{0}\right)^{1 / n}=O\left[\left(N_{n}^{0}\right)^{1 / n}\right]$ or $\left(M_{n}^{0}\right)^{1 / n}=O\left[\left(N_{n}\right)^{1 / n}\right]$. 
Proof. Since $\mathscr{C}_{M}^{*}(I) \equiv \mathscr{C}_{M^{0}}^{*}(I)$ and $\mathscr{C}_{N}^{*}(I) \equiv \mathscr{C}_{N^{0}}^{*}(I)$, the sufficiency of the conditions is obvious. So we prove that they are necessary. We may suppose, without loss of generality, that $I=]-1,1[$ or $]-1, \infty[$ or $\mathbf{R}$. For each $n$, choose an integer $h_{n} \geq n$ such that

$$
\frac{h_{n}^{n}}{S_{M^{0}}(r)} \geq \frac{M_{n}^{0}}{e} .
$$

For $n \geq 2$, set $I_{n}=\left[-1+\left(1 / h_{n}\right), 1-\left(1 / h_{n}\right)\right]$ or $\left[-1+\left(1 / h_{n}\right), h_{n}\right]$ or $\left[-h_{n}, h_{n}\right]$ according as $\left.I=\right]-1,1[$ or $]-1, \infty[$ or $[-\infty, \infty[$. Let $\mathscr{F}$ denote the class of functions $f \in \mathscr{C}^{\infty}(I)$ such that

$$
p_{k}(f)=\sup _{n \geq 0} \max _{x \in I_{k}} \frac{\left|f^{(n)}(x)\right|}{M_{n}^{0}}, \quad k=2,3, \ldots
$$

$\mathscr{F}$ is a Fréchet space with seminorms $\left\{p_{k}\right\}$ and $\mathscr{F} \subseteq \mathscr{C}_{M^{0}}^{*}(I)$. Set

$$
V_{j}=\left\{f \in \mathscr{F}:\left|f^{(n)}(0)\right| \leq j^{n+1} N_{n}^{0}(n \geq 0\}, \quad j=1,2, \ldots .\right.
$$

If $f \in \mathscr{F}, f \in \mathscr{C}_{M^{0}}^{*}(I) \subseteq \mathscr{C}_{N^{0}}^{*}(I)$. Hence $f \in V_{j}$ for some $j$. Thus $\mathscr{F}=$ $\bigcup_{1}^{\infty} V_{j}$. Clearly, $V_{j}$ 's are closed in $\mathscr{F}$. Hence, by Baire's category theorem, there exists a seminorm $p_{r}$, a $\delta>0$ and a $V_{s}$ such that $p_{r}(f) \leq \delta$ implies that $f \in V_{s}$. Set $\alpha=1 / 6 h_{r}$ and let

$$
f(x)=\frac{\delta Z_{h_{n}}(\alpha x)}{2 S_{M^{0}}\left(h_{n}\right)},
$$

where

$$
Z_{n}(x)=(-1)^{[n / 2]} T_{n}(x)+(-1)^{[(n-1) / 2]} T_{n-1}(x),
$$

$T_{n}(x)$ denoting the Chebyshev polynomial of degree $n$ and $[t]$, the integral part of $t$. For $x \in I_{q}, f^{(k)}(x) \equiv 0$ if $k>h_{n}$, and

$$
\left|f^{(k)}(x)\right|=\frac{1}{2} \delta \alpha^{k} \frac{\left|Z_{h_{n}}^{(k)}(\alpha x)\right|}{S_{M^{0}}\left(h_{n}\right)} \leq A_{q} M_{k}^{0}
$$

if $1 \leq k \leq h_{n}$, since

$$
\left|T_{n}^{(k)}(x)\right| \leq(4 n)^{k} e^{4 n e|x|}, \quad x \in \mathbf{R} .
$$

Thus $f \in \mathscr{F}$. If $x \in I_{r}$, then

$$
\left|f^{(k)}(x)\right| \leq \delta \frac{h_{n}^{k}}{S_{M^{0}\left(h_{n}\right)}} \frac{3^{k} \alpha^{k}}{\left(1-\alpha^{2} h_{r}^{2}\right)^{k}} \leq \delta M_{k}^{0}
$$

since (see [10], p. 206) for $-1<x<1$ :

$$
\left|Z_{n}^{(k)}(x)\right| \leq \frac{2.3^{k} n^{k}}{\left(1-x^{2}\right)^{k}} \quad(0 \leq k \leq n) .
$$


Hence $p_{r}(f) \leq \delta$. Then $f \in V_{s}$ and so for every $k \geq 1$,

$$
\frac{1}{2} \delta \alpha^{k} \frac{\left|Z_{h_{n}}^{(k)}(0)\right|}{S_{M^{0}}\left(h_{n}\right)} \leq s^{k+1} N_{k}^{0} .
$$

Since

$$
(n / e)^{k} \leq\left|Z_{n}^{(k)}(0)\right| \leq n^{k}
$$

it follows that

$$
\frac{1}{2} \delta \frac{\alpha^{k}}{e^{k}} \frac{h_{n}^{k}}{S_{M^{0}}\left(h_{n}\right)} \leq s^{k+1} N_{k}^{0} .
$$

Choosing $k=n$ and using (8), we see that, for some $\beta>0$,

$$
\beta^{n} M_{n}^{0} \leq N_{n}^{0} \quad(n \geq 1) .
$$

Thus the first condition is necessary and so also the second.

If we apply this lemma with $N_{n}=M_{n}^{0}$, we get the first half of (7)(a). To prove $(7)(b)$, we need the following:

Lemma III. If $I$ is not an open interval, then $\mathscr{C}_{M}^{*}(I) \subseteq \mathscr{C}_{N}^{*}(I)$ if and only if $\left(M_{n}^{f}\right)^{1 / n}=O\left[\left(N_{n}^{f}\right)^{1 / n}\right]$ or $\left(M_{n}^{f}\right)^{1 / n}=O\left[\left(N_{n}\right)^{1 / n}\right]$.

Proof. Since $\mathscr{C}_{M}^{*}(I) \equiv \mathscr{C}_{M^{f}}^{*}(I)$ and $\mathscr{C}_{N}^{*}(I) \equiv \mathscr{C}_{N^{f}}^{*}(I)$, we need prove only the necessity part of the lemma. Suppose $I=\mathbf{R}_{+}$. Let $I_{h}=[0, h](h \geq 2)$ and let $\mathscr{F}$ denote the class of functions $f \in \mathscr{C}^{\infty}(I)$ such that for each $h \geq 2$,

$$
p_{h}(f)=\sup _{n \geq 0}\left(\max _{x \in I_{h}}\left|f^{(n)}(x)\right| / M_{n}^{f}\right)<\infty .
$$

$\left\{p_{h}\right\}$ is a family of seminorms on $\mathscr{F}$ making $\mathscr{F}$ a Fréchet space. Let

$$
V_{j}=\left\{f \in \mathscr{F}:\left|f^{(n)}(1)\right| \leq j^{n+1} N_{n}^{f} \quad(n \geq 1), \quad j=1,2, \ldots\right\}
$$

Clearly $\mathscr{F}=\bigcup_{1}^{\infty} V_{j}$ and $V_{j}$ 's are closed. Applying Baire's category theorem, we get a seminorm $p_{r}$, a $\delta>0$ and a $V_{s}$ such that $p_{r}(f) \leq \delta$ implies that $f \in V_{s}$. Let

$$
f(x)=\frac{\delta T_{k_{n}}(x / r)}{U_{M^{f}}\left(k_{n}\right)},
$$

where $T_{n}$ denotes the Chebyshev polynomial of degree $n$ and $\left\{k_{n}\right\}$ a sequence of integers chosen such that $k_{n} \geq n$ and

$$
\frac{k_{n}^{2 n}}{U_{M^{f}}\left(k_{n}\right)} \geq \frac{n^{n} M_{n}^{f}}{e^{2}}
$$

$f \in \mathscr{F}$ since $f^{(m)}(x)=0$ for $x \in I_{h}$ and $m>k_{n}$ and, by (9),

$$
\left|f^{(m)}(x)\right| \leq A_{h} M_{n}^{f}, \quad x \in I_{h}, \quad m \leq k_{n} .
$$


Since, for $x \in[-1,1],\left|T_{n}^{(j)}(x)\right| \leq\left(e n^{2} / 2 j\right)^{j}$, we have

$$
p_{r}(f) \leq \frac{\delta}{U_{M^{f}}\left(k_{n}\right)} \sup _{m \leq k_{n}} \frac{k_{n}^{2 m}}{m^{m} M_{m}^{f}} \leq \delta,
$$

Therefore $f \in V_{s}$ and so for each $m \geq 1$.

$$
\delta r^{-m}\left(\frac{2}{e m}\right)^{m} \frac{k_{n}^{2 m}}{U_{M^{f}}\left(k_{n}\right)} \leq s^{m+1} N_{m}
$$

Since $T_{n}^{(j)}(1) \geq\left(2 n^{2} / e j\right)^{j}$. Choosing $m=n$, we get from (11)

$$
\delta r^{-n}\left(\frac{2}{e n}\right)^{n} e^{-2} n^{n} M_{n}^{f} \leq s^{n+1} N_{n}^{f}
$$

Thus $\left(M_{n}^{f}\right)^{1 / n}=O\left[\left(N_{n}^{f}\right)^{1 / n}\right]$ and so also $\left(M_{n}^{f}\right)^{1 / n}=O\left[\left(N_{n}\right)^{1 / n}\right]$.

If $I$ is finite, we may take it to be $[-1,1]$ or $]-1,1]$. Let $\mathscr{B}$ denote the Banach space of functions $f \in \mathscr{C}^{\infty}(I)$ such that

$$
\|f\|_{\mathscr{B}}=\sup _{n \geq 0}\left(\max _{0 \leq x \leq 1}\left|f^{(n)}(x)\right| / M_{n}^{f}\right)>\infty
$$

Clearly $\mathscr{B}$ is a union of the closed sets $V_{j}$, where

$$
V_{j}=\left\{f \in \mathscr{B}:\left|f^{(n)}(1)\right| \leq j^{n+1} N_{n}^{f} \quad(n \geq 1), \quad j=1,2, \ldots\right\}
$$

Arguing as before with $r=(e / 2)$ in (10), we complete the proof.

If $X=\mathscr{C}_{M}^{*}(I) \supseteq \mathscr{C}_{n !}^{*}(I)$, we choose $N_{n}=M_{n}^{d}$ in Lemma III, and get the first half of $(7)(b)$.

The following theorem characterizes the inverse-closed local Carleman classes:

Theorem 2. Let $X=\mathscr{C}_{M}^{*}(I)$. The following assertions are equivalent:

(a) The sequence $A=\left\{A_{n}\right\}$ is almost increasing.

(b) Analytic functions operate on $X$.

(c) $X$ is an inverse-closed algebra.

Here $A_{n}=\left(M_{n}^{0} / n !\right)^{1 / n}$ or $\left(M_{n}^{f} / n !\right)^{1 / n}$ according as $I$ is open or not. 
Proof. (i) Let $X \equiv \mathscr{C}_{M}^{*}(I)$, where $I$ is an open interval which we may suppose, without loss of generality, to be ]-1, 1[ or ]-1, $\infty[$ or $\mathbf{R}$. Let (a) hold. Since $A$ is almost increasing, from (2), we conclude that analytic functions operate on $X$. This trivially implies that $X$ is an algebra. Thus (b) holds.

Suppose that (c) holds. If we choose $f(x)=1+x^{2}$, then $f$ and consequently $f^{-1}$ belongs to $X$ so that $n ! \leq A B^{n} M_{n}(n \geq 1)$. Thus $\mathscr{C}_{n !}^{*}(I) \subseteq X$ and $X \equiv \mathscr{C}_{M^{c}}^{*}(I)$. Moreover, reasoning as in (i) of the proof of Theorem 1 and using the function $f$ constructed there, we see that $\left\{\left(M_{n}^{c} / n !\right)^{1 / n}\right\}$ is almost increasing. But then, by (7), $A$ is also almost increasing. Thus (c) holds.

(ii) Let $X \equiv \mathscr{C}_{M}^{*}(I)$, where $I$ is not open. Then $X \equiv \mathscr{C}_{M^{f}}^{*}(I)$. Here we take $A_{n}=\left(M_{n}^{f} / n !\right)^{1 / n}(n \geq 1)$. As in (i), (a) implies (b) and (b) implies (c). We only need show that (c) implies (a).

Without loss of generality, we may suppose that $I=[0,1]$ or $] 0,1]$ or $\mathbf{R}_{+}$. Since $X$ is inverse-closed and $f \in X$, where $f(x)=1+x$, it follows that $f^{-1} \in X$ so that $X \supseteq \mathscr{C}_{n !}^{*}(I)$. But then, by Lemma I, $X \equiv \mathscr{C}_{M^{d}}^{*}(I)$. Hence the function $f$ constructed in (ii) of the proof of Theorem 1 or its restriction is in $X$ and so also its inverse $f^{-1}$. From this point on the same proof with obvious modifications goes through and we conclude that $\left\{\left(M_{n}^{d} / n !\right)^{1 / n}\right\}$ is almost increasing. But then, by (7), $A$ is almost increasing.

\section{REMARKS}

We make a few concluding remarks.

$1^{\circ}$. The following theorem which completes Theorem A characterizes inverse-closed Carleman and local Carleman algebras defined by log-convex sequences $M$.

Theorem 3. Let $X=\mathscr{C}_{M}(I)$ or $\mathscr{C}_{M}^{*}(I)$, where $M$ is log-convex. The following assertions are equivalent.

(a) $\left\{\left(M_{n} / n !\right)^{1 / n}\right\}$ is almost increasing.

(b) Analytic functions operate on $X$.

(c) $X$ is an inverse-closed algebra.

Proof. It suffices to note that (c) implies (a) as in (i) of the proof of Theorem 1 since, by trivial modifications, the characteristic function constructed there becomes such a function for any class $\mathscr{C}_{M}(I)$ or $\mathscr{C}_{M}^{*}(I)$ with the desired properties.

$2^{\circ}$. Theorems 1 and 2 characterize all inverse-closed Carleman and local Carleman algebras if we note that $\mathscr{C}_{M}(I) \equiv \mathscr{C}_{M}(\bar{I})$ and that for finite $I$, $\mathscr{C}_{M}(I) \equiv \mathscr{C}_{M}(\bar{I}) \equiv \mathscr{C}_{M}^{*}(I)$.

$3^{\circ}$. If we repeat the proof of Theorem 1 for the class $X \equiv \mathscr{C}_{M}^{2 \pi}([0,2 \pi])$, replacing the function $f$ used there by the function

$$
f(x)=\sum_{j=1}^{\infty} \frac{e^{i\left[r_{j}\right] x}}{2^{j} T_{M^{c}}\left(r_{j}\right)},
$$


where $[t]$ denotes, as usual, the integral part of $t$, we conclude in response to the question raised by $\mathrm{W}$. Rudin [11] that $X$ is inverse-closed if and only if $\left\{\left(M_{n}^{c} / n !\right)^{1 / n}\right\}$ is almost increasing.

\section{REFERENCES}

1. T. Bang, Om quasianalytiske funktioner, Nyt Nordlisk Forlag, Copenhagen, 1946.

2. J. Boman and L. Hörmander, Classes of infinitely differentiable functions, (mimeographed notes) (Swedish), Stockholm, 1962.

3. J. Bruna, On inverse-closed algebras of infinitely differentiable functions, Studia Math. 69 (1980), 61-67.

4. H. Cartan, Solution d'une problème de Carleman pour un intervalle fermé fini, C. R. Acad. Sci. Paris 208 (1939), 716-718.

5. __ Sur les classes de fonctions définies par des inégalités portant sur les dérivées successives, Herman, Paris, 1940.

6. A. Gorny, Contributions à l'études des fonctions dérivables d'une variable réele, Acta Math. 71 (1939), 317-358.

7. J.-P. Kahane, Séries de Fourier absolument convergentes, Springer-Verlag, Berlin-HeidelbergNew York, 1970.

8. P. Malliavin, Calcul symbolique et sous-algèbres de $L^{1}(G)$, Bull. Soc. Math. France 87 (1959), 181-186.

9. A. Mallios, Topological algebras. Selected topics, North-Holland Math. Studies, 124, Amsterdam, 1986.

10. S. Mandelbrojt, Séries adhérentes, régularisatiosn des suites, applications, Gauthier-Villars, Paris, 1952.

11. W. Rudin, Division in algebras of infinitely differentiable functions, J. Math. Mech. 11 (1962), 797-809.

12. J. A. Siddiqi, Sur les classe de fonctions différentiables fermées par passage à l'inverse, C. R. Acad. Sci. Paris 305 (1987), 9-12.

Département de Mathématiques et de Statistique, Université Laval, Cité UniversiTAIRE, Québec, P.Q. G1K 7P4 CANADA 